\title{
KoenraAd Kuiper
}

\section{Phraseologie aus der Sicht der generativen Grammatik}

\begin{abstract}
Innerhalb der generativen Theorien in Chomskys Tradition spielt das Lexikon während der letzten zwanzig Jahre eine zunehmend wachsende Rolle. Dies ist nicht zuletzt auf das Projektionsprinzip zurückzuführen. Es besagt, dass lexikalische Einheiten ihre semantischen und syntaktischen Grundzüge in allen relevanten Repräsentationen in der Grammatik beibehalten (Chomsky 1981). Die Grammatik wird in dieser Sichtweise als lexikalisch getrieben aufgefasst. Es ist deshalb auffallend, dass phraseologische Einheiten in der generativen Grammatik nur eine untergeordnete Rolle spielen, schlicht deshalb weil solche Einheiten als lexikalisch betrachtet werden. Dieser Vortrag geht zuerst auf einige theoretische Debatten ein, in denen der Status von phraseologischen Einheiten behandelt wird. Sodann wird gefragt, welche Funktion diese innerhalb einer generativen Sprachtheorie einnehmen können. Im Zentrum steht eine generative Theorie des Lexikons und das Problem, wie eine solche Theorie möglicherweise die syntaktischen Eigenschaften phraseologischer Einheiten erklären könnte.
\end{abstract}

\section{Vorbemerkungen}

Ich bin gebeten worden, einen kurzen Abriss der generativen Grammatik zu geben und darüber zu sprechen, welche Rolle die Phraseologie in den Sprachtheorien Chomskys spielt. Dieses Auftrags könnte ich mich sehr leicht entledigen. Wenn man nämlich die fünfzigjährige Entwicklung der generativen Theorie betrachtet, und sich fragt, was man von Chomskys Theorien in Bezug auf den Bereich Phraseologie, so wie er im Deutschen Sprachbereich verstanden wird, sagen kann, dann kann man nur antworten, gar nichts. Damit könnte ich meinen Vortrag beenden.

Ich möchte aber eine kleine Wendung machen und statt der Phraseologie die phraseologischen Einheiten selbst aus der Sicht der generativen Grammatik betrachten. Zu diesem Thema kann mehr gesagt werden.

Ich werde zunächst einen Abriss der Entwicklung der generativen Grammatik vortragen - soweit sie phraseologische Einheiten betrifft. Dann werde ich eine kurze Übersicht über einige der syntaktischen Eigenschaften phra-

1 Ich möchte den Folgenden für ihre Hilfe danken: Otto Dann, Anandi Eichenberger, Martin Everaert, Dani Schreier, Hannelore van der Watering, dem Netherlands Institute for Advanced Study, der University of Canterbury und dem IDS für die Einladung, die es mir ermöglicht hat, bei der 39. Tagung einen Vortrag zu halten. 
seologischer Einheiten geben und fragen, wie sie, theoretisch gesehen, in einer modernen generativen Grammatik behandelt werden können.

Als Fachausdruck ist ,generativ' zweideutig. Auf der einen Seite bezeichnet er eine Theorie mit mathematischer Form. Das Ziel einer solchen Theorie ist davon empirische Konsequenzen ableiten zu können (Chomsky 1975, S. 86). Auf der anderen Seite wird der Ausdruck manchmal für die Theorien der Chomsky'schen Sprachauffassung gebraucht. Nicht alle generativen Theorien im ersten Sinn sind auch im zweiten Sinn generativ. Zum Beispiel: eine head-driven phrase structure grammar (Pollard/Sag 1988) oder eine lexical functional grammar (Bresnan 1982) sind im ersten Sinn generativ, nicht aber im zweiten.

Die Theorien Chomskys sind zudem in den vergangenen fünfzig Jahren durch eine Reihe von Entwicklungen mehrmals modifiziert worden. Die Standard-Theorie (Chomsky 1965) änderte sich in den Prinzipien und Parameter Rahmen (Chomsky 1981) und von dort aus ins Minimalismusprogramm (Chomsky 1996). Jede dieser Theorien hat sich wiederum selbst durch einen Stufenwandel geändert.

\section{Zum Status phraseologischer Einheiten innerhalb der generativen Grammatik}

\subsection{Einführung}

Um zu verstehen, welche Bedeutung phraseologische Einheiten in der generativen Grammatik haben, sollte man sich zunächst deren Grundanliegen vor Augen führen. Sie wurden bereits am Anfang der generativen Grammatik benannt (Chomsky 1965; Chomsky 1975). Eine linguistische Theorie muss erstens ,observatorisch adäquat' sein, das heißt, sie muss in der Lage sein, eine Beschreibung von den Wortfolgen der Sätze einer Sprache zu geben. Zweitens sollte eine Theorie, deskriptiv adäquat' sein, das heißt, sie sollte eine Verbindung herstellen zwischen den Beschreibungen individueller Sätze einer Sprache und der verinnerlichten Grammatik des Sprechers. Schließlich muss eine generative Sprachtheorie erklärungsfähig sein, das heißt, sie sollte darlegen können, wie sich Kinder so schnell und gleichmäßig und mit so wenig Spracherfahrung eine Sprache aneignen. Dabei geht man von der Grundannahme aus, dass von den vielen Grammatiken, die ein Kind sich aneignen könnte, nur eine Grammatik auch tatsächlich erworben wird. Dies geschieht auf Grund des Umstandes, dass alle Menschen eine Universalgrammatik besitzen. Der Spracherwerb ist nur möglich, weil die Universalgrammatik die Vielfalt möglicher Grammatiken menschlicher Sprachen einschränkt. Die Theorie von der Universalgrammatik ist demzufolge in ein System menschlicher Erbanlagen eingebettet. Die Universalgrammatik, also wie genau Kinder Sprache erwerben, ist und bleibt das wichtigste Ziel der generativen Grammatik.

Im Rahmen von Prinzipien und Parameter nimmt die Universalgrammatik folgende Gestalt an. Es gibt eine Reihe universaler Prinzipien, die für alle 
menschlichen Sprachen Anwendung finden. Für jedes Prinzip gibt es auch einen Parameter, der besagt, wie Sprachen sich unter diesem Prinzip von einander unterscheiden. Der „Kopf" einer syntaktischen Konstruktion, zum Beispiel, befindet sich links vom Komplement oder rechts von ihm. Deshalb werden Sprachen als Kopf-erst oder Kopf-letzt bezeichnet. Im Deutschen kommt das Verb nach seinem Komplement, im Englischen aber das Komplement nach dem Verb. Es gibt Sprachen, in denen das grammatische Subjekt nicht ausgedrückt sein muss, so im Italienischen, und andere Sprachen, wo das unmöglich ist, wie im Englischen. Im Italienischen kann Ho telefonato (,habe telefoniert') ein Satz sein, im Deutschen kann es dies nicht. Der Pro-drop Parameter, der für diese Möglichkeiten sorgt, ist ein Parameter aller Sprachen. Durch ihr Aufwachsen in einer Sprachgemeinschaft können Kinder herausfinden, wie die Sprache ihrer Umgebung jeden Parameter benutzt. Es ist also generell so, dass das Italienische Sätze ohne Subjekt zulässt, während das Deutsche dies nicht erlaubt.

Eines dieser Prinzipien ist auch für phraseologische Einheiten wichtig. Das Projektionsprinzip besagt (ungefähr), dass syntaktische Eigenschaften lexikaler Einheiten in der Syntax nicht umgeschrieben werden können. Das bedeutet für Wörter, dass in der Struktur eines Satzes ein Verb transitiv bleibt. Das ist eine syntaktische Eigenschaft des Verbs und nicht zu ändern (Chomsky 1981, S. 29). Für phraseologische Einheiten, die natürlich auch lexikalische Einheiten sind, wirft das Projektionsprinzip die Frage auf, wie deren syntaktische Eigenschaften in die Struktur eines Satzes projektiert werden. Darauf wird später einzugehen sein.

Zunächst noch zu weiteren Problemen. Chomsky meint, dass eine Sprachtheorie nur über eine interne Grammatik (I Grammatik), also die Grammatik eines Sprechers und nicht die einer Sprache, etwas aussagen kann (Chomsky 1986b). Das gilt auch für das Lexikon. Generative Theorien interessieren sich nicht für die Wörter einer Sprache, jedoch für die Wörter im internen Lexikon eines Sprechers. Externe Sprachen sind, nach Meinung Chomskys, unzusammenhängend. Für diejenigen, die mit phraseologischen Wörterbüchern zu tun haben, ist das ein Problem. Beschreibungen des phraseologischen Wortschatzes einer ganzen Sprache oder einer Fachsprache, oder stilistische Untersuchungen auf phraseologischem Gebiet sind wissenschaftlich nicht verantwortbar. Das betrifft den größten Teil phraseologischer Untersuchungen.

Weiterhin befasst sich die generative Grammatik nur mit der Kerngrammatik eines Sprechers, denn die interessanten Eigenschaften einer Innengrammatik können von der Universalgrammatik abgeleitet werden (Chomsky 1981). Obwohl das Lexikon in der generativen Grammatik eine prominente Rolle spielt, wird wenig darüber gesagt, wie die Wörter einer Innengrammatik von der Universalgrammatik abgeleitet werden können.

Chomsky meint, dass Konzepte universal seien, aber vielleicht nicht alle Konzepte. Weil es möglicherweise Konzepte gibt, die man nicht zu lernen 
hat, muss man auch nicht unendlich viele Wörter erwerben. Wenn man den phraseologischen Wortschatz betrachtet, kann man kaum verstehen, dass eine jede phraseologische Einheit universal sein soll. Phraseologische Einheiten befinden sich deshalb wohl nicht in der Kerngrammatik eines Sprechers, sondern an der Peripherie, wie Chomsky das nennt. Daher sind sie aus der Sicht der generativen Grammatik nicht interessant (Becker 1975).

Wir wenden uns nun dem Minimalismusprogramm zu. Hier finden wir, dass die bekannten Ebenen der Tiefen- und der Oberflächenstruktur verschwunden sind. Nur die Ebene der logischen Form und der phonetischen Form bleiben bestehen, ganz im Sinne von de Saussure, der die Sprache als einen Zusammenhang von Laut und Bedeutung versteht (de Saussure 1959). Der Grund für das Aufgeben der Syntax mit zwei Ebenen ist naheliegend: sie sind aus konzeptuellen Gründen nicht nötig. Das erinnert an eine Frage der generativen Semantiker in den 60er Jahren: ,Ist die Annahme einer Tiefenstruktur eigentlich nötig?' Es scheint, dass die Antwort heute ,nein ' lauten muss. Lexikale Einheiten bleiben wichtig für die Theorie, weil sie Strukturinformationen darüber beinhalten, wie die syntaktische Form eines Satzes aufgebaut wird. Das Projektionsprinzip besagt, dass eine neue Fusionsregel Wörter mit Hilfe der syntaktischen Möglichkeiten der Universalgrammatik zusammensetzt. Die Baumstruktur wird von unten nach oben aufgebaut. Die Struktur ist in allen Sprachen dieselbe. Syntaktische Bewegungen können dann zwischen der logischen Form und der phonologischen Form vermitteln. Dementsprechend wird das Lexikon nur zuunterst in der Baumstruktur nachgeschlagen. Konstituenten können nur weiter nach oben gelangen, indem sie durch syntaktische Bewegungen nach links oder nach oben verschoben werden. Andere Bewegungen sind nicht möglich. Phraseologische Einheiten scheinen also im Minimalismusprogramm keine zentrale Rolle zu spielen.

\subsection{Was sagt die Standardtheorie über phraseologische Einheiten?}

Idiome wurden in der generativen Grammatik zum ersten Mal behandelt, und zwar auf den letzten Seiten von Chomskys Aspects (Chomsky 1965), wo er sich mit der Morphologie befasst. „Nehmen Sie zum Beispiel Phrasen wie ,take for granted", die sehr oft im Englischen vorkommen," heißt es dort. „Aus der Sicht der Semantik und der Wortfolge scheint diese Phrase eine Einheit zu sein. Deshalb sollte sie sich im Lexikon als eine Einheit mit eigenen syntaktischen und semantischen Eigenschaften benehmen. Aber auf der anderen Seite ist sie, in Bezug auf Transformationen und Morphologie, eine Verb plus Komplement Struktur. Hier haben wir also eine lexikale Einheit mit einer reichen inneren Struktur" (Chomsky 1965, S. 190). Zwei zentrale Eigenschaften sind daher zu erklären. Idiome sind in syntaktischer Hinsicht komplex, semantisch und von ihrer Distribution her gesehen jedoch einfach. Man kann hinzufügen, dass diese Eigenschaften nicht immer gemeinsam auftreten. Feste Kollokationen können, allein aus der Sicht ihrer 
Distribution, lexikalische Einheiten sein. Aber eine Einheit, die idiomatisch ist, muss auch als eine einheitliche Wortfolge vorkommen. Wie „einheitlich“ solche Einheiten sind, bleibt eine interessante Frage. Was Chomsky dazu zu sagen hat, ist eigentlich nicht mehr als eine Definition. Es ergeben sich phraseologische, das heißt lexikalische Einheiten, die sich wie syntaktische Einheiten verhalten. Ein Teil dieser Einheiten sind Idiome, weil sie semantisch nicht-kompositionell sind.

Weinreich hat etwas später die Problematik Chomskys weitergeführt (Weinreich 1969). Er beschreibt viele Arten der „Nicht-Kompositionalität“ bei Zusammensetzungen und Idiomen und ist der Meinung, dass „NichtKompositionalität ein extremes Beispiel für die Kontextspezialisierung einer Bezeichnung ist, die durch eine Reihe von Eigenschaften definiert wird, die auch anderswo zu finden sind" (Weinreich 1969, S. 224). Weinreich hat außerdem Wichtiges über die semantische Motivierung von Idiomen zu sagen. Er glaubt, dass diese Motivierung nur post factum betrachtet werden kann (Weinreich 1969, S. 259). Wie nicht-kompositionell ein Idiom ist, hat Auswirkungen darauf, wie weit es durch Transformationen verändert werden kann. Aber die Beziehungen sind nicht kausal - einfach deshalb, weil es nicht in jedem Fall möglich ist zu sagen, wie sie aussehen und funktionieren sollen. Weinreich schlägt zudem vor, eine generative Grammatik solle ein Idiomenverzeichnis ins Lexikon übertragen. Ein solches Verzeichnis müsste für jedes Idiom angeben: 1. die Wörter im Idiom, 2. ihre syntaktische Struktur, und 3. ihre Bezeichnung. Viertens sollte auch angegeben werden, welche Transformationen für das Idiom obligatorisch, möglich oder nicht möglich sein sollen. Die Grammatik selbst generiert Sätze und vergleicht Phrasen und Sätze des Outputs mit dem Idiomenverzeichnis. Wenn sich in diesem Verzeichnis ein ähnlicher Ausdruck befindet, kann die Grammatik auch das Idiom selbst generieren, indem sie es innerhalb des geeigneten syntaktischen Rahmens einführt.

Die Frage, ob Idiome nicht-kompositionell sind, war Auslöser für eine Auseinandersetzung während der Linguistikkriege der Sechzigerjahre (Harris 1995). Chafe (1968) behauptete, dass vier Tatsachen über Idiome fatal seien für Chomskys Theorie, Idiome seien nicht-kompositionell. Sie könnten nicht immer und auf alle Weisen transformiert werden. Einige seien, syntaktisch gesehen, mangelhaft und kämen mit großer Häufigkeit in Texten vor. Da die Theorie einer generativen Semantik diese Probleme nicht hat, sei diese eine adäquatere Theorie. Die Antwort auf diesen Vorschlag kam von Fraser (1970): Es gäbe zwei Probleme mit Chomskys Theorie: 1. wie können Idiome in die Tiefenstruktur eines Satzes eingeführt werden und 2. wie könne man die transformationellen Eigenarten in der Theorie Chomskys erklären. Textfrequenz ist ein Problem für die Sprachtheorie an sich und als solches nicht durch eine grammatische Theorie zu erklären. Fraser (1970, S. 27) schlug vor, dass Idiome mit eigener Struktur in einen Strukturbaum eintreten. Das warf das Problem auf, dass viele Idiome leere Stellen haben, 
in welche die Konstituenten erst später eintreten müssen (Fraser 1970, S. 28-9). Zum Beispiel das englische Idiom put something in order: something ist hier eine leere Stelle, in die eine Nominalphrase eingefügt werden muss. Ein weiteres Problem: einige dieser leeren Stellen haben Referenzhinweise auf andere Nominalphrasen. Solche Hinweise sind, theoretisch gesehen, nicht voraussagbar. Im englischen Idiom to lose one's mind ist one koreferent mit dem Subjekt des Satzes. Aber in break someone's heart kann someone nicht ko-referent mit dem Subjekt des Satzes sein. Diese Eigenschaften des Idioms müssen im Lexikon aufgeführt sein, um von dort aus ihre Wirkung auf die Syntax des Satzes zu haben (Fraser 1970, S. 31). Ungrammatische Idiome werden im Lexikon mit einer vergleichbaren syntaktischen Form aufgeführt, weil diese Form für die Phonologie benötigt wird, aber nicht für die Syntax (Fraser 1970, S. 31).

Das Problem der syntaktischen Bewegungen von Idiomen wird von Fraser eigentlich nicht gelöst. Er behauptet, jedes Idiom gehöre zu einer Stufe. Diese Treppenstruktur sage, welche Bewegungen auf jeder Stufe möglich seien. Unten können Idiome keine Bewegungen ausführen, während ihnen oben alle Möglichkeiten offen stehen. Wenn ein Idiom zu einer Stufe gehört, können alle Transformationen auf dieser Stufe und auf den darunter liegenden stattfinden (Fraser 1970, S. 36-41). Es gibt jedoch wenig Evidenz für die Existenz einer solchen Treppenstruktur. Sie wird demzufolge auch in der generativen Theorie nicht weiter behandelt.

Dass es eine Beziehung zwischen der syntaktischen Teilbarkeit und der semantische Form eines Idioms gebe, wird von Nunberg et al. (1994) vorgeschlagen. Einen kritischen Überblick dieser Theorie finden wir bei Dobrovol'skij (2000).

In den frühen siebziger Jahren haben Chomsky (1970) und, zum Teil, auch Halle (1973) eine Modifizierung der Standardtheorie, namentlich des Lexikalismus, durchgeführt. Bei Anwendung des Lexikalismus sei es möglich, nicht nur syntaktische Regeln, sondern auch im Lexikon befindliche morphologische Regeln in eine Grammatik einzuführen. Der Vorschlag Halles hat etwas gemeinsam mit den Ideen Weinreichs, doch aufgrund der Vorschläge Jackendoffs fanden phraseologische Einheiten schließlich Platz in der generativen Theorie (Jackendoff 1975). Diese Vorschläge sind wichtig, weil sie das erste und wohl auch interessanteste Bild davon geben, wie man in eine generative Theorie phraseologische Einheiten einbauen kann. Jackendoff ist der Meinung, dass morphologische und semantische Eigenschaften morphologisch komplexer Wörter von einander unabhängig sind. Zum Beispiel: Sammlung ist das Resultat von sammeln und nicht das Sammeln selbst, weil eine Ausführung das Ausführen selbst ist. Die verschiedenen Regeln müssen deshalb für alle Wörter miteinander verbunden werden. Wenn beide regelmäßig sind, gibt es eine Regel für die Morphologie eines Wortes und eine andere Regel für dessen Bedeutung. Jedes Wort erhält volle Repräsentation im Lexikon. Die Regeln des Lexikons zeigen, was von der Repräsentation 
zu lernen ist. Diese Vorschläge können sich an Idiome anpassen. Idiome werden im Lexikon mit allen ihren Eigenschaften angegeben. Was an den Idiomen regelmäßig ist, kann durch die Regeln der Syntax als überflüssig angesehen werden, und was übrig bleibt, muss gelernt werden. Wenn die Bezeichnung nicht-kompositionell ist, kann die Semantik sie nicht voraussagen. Wenn die syntaktischen Bewegungen einer phraseologischen Einheit eigenartig sind, kann die Syntax der Sprache sie nicht voraussagen. Aber mit festen Kollokationen verhält es sich meist so, dass nur die Auswahl der Wörter eigenartig ist. Andere linguistische Eigenschaften sind regelmäßig. Die Theorie Jackendoffs sagt, dass für solche Einheiten nur die Folge der Wörter zu lernen ist.

Man kann sich nun fragen, ob und wie diese Theorie sich um den Spracherwerb bemüht. Sie versucht, explanatorisch adäquat zu sein. Morphologisch komplexe Wörter und phraseologische Einheiten sind leichter zu lernen, denn sie enthalten so viel überflüssige Information. Zum Beispiel ist vieles von dem, was in einer phraseologischen Einheit repräsentiert werden muss, auch anderswo im Lexikon repräsentiert; denn die Wörter in einer phraseologischen Einheit haben ihren eigenen Platz im Lexikon. Phraseologische Einheiten werden, wie Wörter, mit allen ihren Eigenschaften im Lexikon angegeben. Es gibt kein spezielles Verzeichnis der Idiome.

Wichtig ist in dieser Hinsicht die Reihenfolge im Lexikon. Wenn ein kompliziertes Wort oder ein komplizierter Ausdruck nur durch Bezugnahme auf andere lexikale Einheiten leicht zu lernen ist, müssen diese Einheiten erst gelernt werden, um ein optimales Resultat zu erhalten. Das ist, intuitiv betrachtet, offensichtlich. Je mehr komplexe lexikalische Einheiten man lernt, desto leichter ist es weitere zu lernen, denn die Eigenschaften der weiteren Einheiten stützen sich auf das, was schon im Lexikon vorhanden ist.

\subsection{Prinzipien und Parameter}

In der Prinzipien- und Parametertheorie ist ein Wandel von der Grammatik einer Sprache zu den Regeln der Universalgrammatik zu beobachten, und zwar in Bezug auf die Beschränkungen von grammatischen Regeln und möglichen Grammatiken. Deshalb werden phraseologische Einheiten selten behandelt. Dennoch bleibt, was Chomsky in Aspects (1965) gesagt hat, wichtig. Phraseologische Einheiten sind lexikalische Einheiten mit syntaktischer Form. Ihre Einheitlichtkeit gibt entweder Probleme auf oder wird als Kriterium in grammatischen Argumenten verwendet.

Hier gibt es allerdings ein Problem: Es gibt phraseologische Einheiten, die zwei Passivkonstruktionen erlauben: ein Innenpassiv und ein Außenpassiv. Im Englischen zum Beispiel kann man His achievements were taken account of und auch Account was taken of his achievements sagen. Im ersten Beispiel wird die phraseologische Einheit als Verb mit Objekt betrachtet. Im zweiten Beispiel wird als Objekt nur das Objekt von take behandelt. Um die phraseologische Einheit als Verb fungieren zu lassen, wird die ganze Struk- 
tur als Verb re-analysiert. Re-analysierung besagt, dass eine phraseologische Einheit als Wort und als Phrase betrachtet werden kann. Diese Behauptung ist aber nicht unproblematisch. Das wird klar in der Kritik von Levine (1984) bezüglich des weiteren Gebrauchs von Re-analysierung in Chomskys Analysen (Chomsky 1981, S. 313). Levine zeigt, dass Phrasen nicht als Wörter anzusehen sind, weil sie sich in mancher Hinsicht nicht als Wörter verhalten. Für diesen Beweis benutzt er syntaktische Theorien der P und P Theorie, die zeigen, dass die re-analysierten Einheiten sich als syntaktische und nicht als wörtliche Einheiten verhalten.

In der weiteren Entwicklung der P und P Theorie kommen phraseologische Einheiten meistens als Idiome vor. Sie werden für eine ihrer primären Eigenschaften benutzt. Man nimmt an, dass sie Einheiten darstellen, die aus dem Lexikon als ununterbrochene Wortketten in die Tiefenstruktur der Syntax gelangt sind. Sollten sie irgendwo unterbrochen werden, dann wird angenommen, das sei das Resultat einer syntaktischen Bewegung. Zum Beispiel: im Deutschen und Niederländischen ist es normal, dass aufgrund der Regel „das Finitum voranstellen“ ein Verb mit Zeitform vom letzten Platz im Satz auf den zweiten Platz im Hauptsatz verschoben wird. Das gilt auch für Idiome. Zum Beispiel in Nebensätzen, wie Ich sah, dass sie von der Spur abkamen, wird von der Spur abkommen an der letzten Stelle eingeführt. Aber mit der Zeitform im Hauptsatz, wie Sie kamen gestern Mittag von der Spur $a b$, ist das Verb von den anderen Wörtern des Idioms getrennt. Man nimmt allgemein an, dass das durch eine syntaktische Bewegung geschieht.

(Es sei auch betont, dass Idiome, wenn man sie auf diese Weise als Argument benutzt, nur einen Teil der Beweise darstellen, auf die sich die folgenden Hypothesen stützen.)

\section{Verben des Erscheinens}

Idiome gelten als Beweis für die Theorie, dass Verben wie scheinen, sogenannte „raising verbs", in der Tiefenstruktur einen Satz als Objekt haben; das Subjekt kann dann entweder ein ,leeres" Wort wie es sein oder das Subjekt des Objektsatzes wird zum Subjekt des Hauptsatzes erhoben. Zum Beispiel kann man Es scheint, dass die Welt zum Teufel geht oder Die Welt scheint zum Teufel zu gehen sagen. Zum Teufel gehen ist im ersten Satz idiomatisch; im zweiten ist das Subjekt von der Verbalphrase getrennt, es ist aus dem Nebensatz zum Subjekt des Hauptsatzes erhoben worden.

\section{Die „Verbphrase-Innensubjekt“ Hypothese}

Diese Hypothese besagt, dass das grammatische Subjekt sich zuerst in der Verbphrase befindet und erst später durch eine Bewegung nach oben in den Strukturbaum gelangen kann. In seinem Buch Barriers (Chomsky 1986a) baut Chomsky das X-quer-Schema von 1970 aus. Die X-quer-Theorie geht davon aus, dass Strukturbäume in allen Sprachen gleiche Eigenschaften ha- 
ben. Jeder „Baum“ hat einen „Kopf“. Die syntaktischen Eigenschaften des „Kopfes“ projektieren hinauf bis zur maximalen Projektion, und es gibt drei Ebenen vom Kopf bis zur maximalen Projektion. Es gibt nur vier mögliche Positionen in einem Strukturbaum: Kopf, Komplement, Spezifikator und Adjunkt. 1986 versuchte Chomsky, dieses Schema nicht nur für Phrasen, sondern auch für ganze Sätze einzuführen. Dabei zeigte sich, dass es nicht nur lexikale Köpfe gibt, sondern auch Funktionsköpfe, nämlich I (Abkürzung für Flexionselement) und $\mathrm{C}$ (Abkürzung für Komplementierer) (Chomsky 1986a). In der Oberflächenstruktur ist das Subjekt der Spezifikator von IP, und IP die maximale Projektion von I. Einige Wissenschaftler, z. B. Koopman und Sportiche (1991), behaupteten, das Subjekt leite sich, wie bei den ,raising verbs“, vom Spezifikator des VPs ab. Idiome kommen also wiederum ins Spiel: man braucht Idiome, die ein Subjekt enthalten, deren Tempus aber nicht fixiert ist, zum Beispiel ein Idiom wie Mir raucht der Kopf. Das Tempus ist hier nicht fixiert, alles andere ist aber Teil des Idioms und damit fixiert. Da dieses Idiom das Tempus nicht einschließt, versucht man, dessen Struktur lediglich als VP mit Innensubjekt zu erklären. Das heißt, das Subjekt ist im VP und wird später erst erhoben. Das kann man im Deutschen nur schwer darstellen, aber bei einem englischen Idiomen wie Much water is passing/could have been passing under the bridge scheint es, dass das Idiom nur Water pass under the bridge umfasst. Weil das Tempus sich höher im Strukturbaum befindet, gelangt Water nur durch eine syntaktische Bewegung an die linke Seite des Tempus (I).

\section{Verben mit zwei Objekten}

Die X-quer-Theorie behauptet, dass Strukturbäume immer nur zwei Zweige unter einem Knotenpunkt haben, deshalb sind Verben wie geben problematisch. Geben hat drei Argumente. Man gibt jemandem etwas. Das VP schließt das Verb und zwei Argumente ein. Das ergibt den folgenden Strukturbaum für VP:

Bild 1.

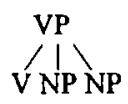

Der Knotenpunkt VP hat jedoch drei Zweige!

Larson (1988) und andere behaupteten, das indirekte Objekt stehe näher zum Verb und deshalb sollte der Strukturbaum folgendermaßen aussehen:

Das Verb wird hier durch eine syntaktische Bewegung zu einem höheren $\mathrm{V}$ erhoben.

Im Englischen werden Idiome als Evidenz für diese Theorie ins Spiel gebracht. Die fixierten Teile von Idiomen wie take NP to the cleaners oder car- 
Bild 2.

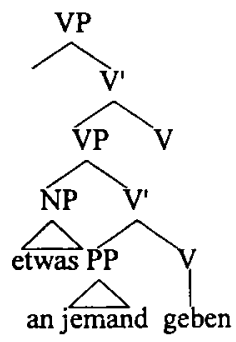

ry NP to extremes, sind durch eine nicht fixierte NP von einander getrennt. Die Analyse Larsons setzt aber die Teile als eine ungebrochene Reihe nebeneinander. Sobald das V nach oben wandert, kommt im Englischen die leere NP zwischen V und PP: die Trennung ist ein Phänomen der Oberfläche.

\section{Sind Subjekt und Objekt als Argumente ähnlich?}

Es ist bekannt, dass jeder „Kopf“ einer Phrase, wie Verben und Präpositionen, zu einer oder mehreren Subkategorien gehört. Es gibt Verben mit und Verben ohne Objekte, das heißt, es gibt transitive und intransitive Verben. Präpositionen können gleichfalls Objekte haben oder auch nicht. Subkategorien werden nie aufgrund des Subjekts erkannt. Ein zentrales Argument von Marantz (1984) in Bezug auf die Asymmetrie von Subjekt und Objekt ist die Behauptung, dass Idiome mit Subjekt und Objekt, in denen das Subjekt fixiert ist, auch das Objekt fixieren jedoch nicht umgekehrt. Dies wiederum hat zu tun mit einer unausgesprochenen Theorie darüber, wie fest die Konstituenten eines Idioms miteinander verbunden sind. Auch hier gibt es kaum empirische Forschungen darüber, ob diese Behauptung zutrifft. Everaert und Kuiper (1996) zeigen, dass es Idiome gibt, die Marantzes Theorie widerlegen, zum Beispiel Hanging is too good for NP und Life passed NP by. (Ob es solche Beispiele auch im Deutschen gibt, weiß ich nicht.)

\subsection{Minimalismus}

Minimalismus ist keine Theorie, sondern ein Programm. Was es als Programm über phraseologische Einheiten zu sagen hat, ist deshalb nicht bekannt. Viel ist hier noch zu tun, und vieles ist umstritten. Die Regel „Merge“ (d.h. zusammensetzen) hat zur Folge, dass das Lexikon ,unten“ und so früh wie möglich eintreten muss, weil Strukturbäume nur auf der Basis von syntaktischen Informationen, die aus dem Lexikon stammen, aufgebaut werden können. So funktioniert das Projektionsprinzip im Minimalismusprogramm. Phraseologische Einheiten haben eine syntaktische Form. Wie das im Minimalismus aber projektiert wird, scheint mir eine offene Frage zu sein.

Idiome werden jedoch auch in Minimalismustheorien als Daten benutzt. Wie das Ende der Tiefenstruktur, zuerst von Generativsemantikern in den 
60er Jahren angekündigt und jetzt vom Minimalismusprogramm übernommen, ist auch das Minimalismusprogramm zurückgekehrt zu dem syntaktischen Bild einer Wortbezeichnung, wie das zuerst von Generativsemantikern vorgeschlagen wurde (Hale/Keyser 1998).

1. Richards (2001) benutzt die angeblich ungebrochene Struktur der Idiome als eine Unterstützung für die Theorie, dass Mary gave Susan the boot und Susan got the boot from Mary miteinander syntaktisch verwandt sind. Was sie gemeinsam haben, ist ein Teil der idiomatischen Bedeutung, nämlich HAVE the boot. Geben ist zu verstehen als jemanden VERURSACHEN etwas zu HABEN und erhalten ist zu verstehen als WERDEN zu HA$B E N$. Diejenigen mit guten und langen Erinnerungen an die Geschichte der generativen Grammatik werden sich hier vielleicht an die Diskussion über kill und cause to die erinnert fühlen.

2. McGinnis (2002) lenkt das Interesse auf die Bedeutung von Idiomen. Sie meint, dass die Aktionsarten der Idiome immer kompositionell sind. Alle Verben haben Aktionsarten: Was passiert, kann dauern oder nicht usw. Das hat Konsequenzen für die Syntax. McGinnis behauptet, es gebe zwei Arten von Bedeutung: strukturelle Bedeutung, die in die Syntax projektiert wird, und eigenartige Bedeutung, die nicht projektiert wird. Idiome haben von $\mathrm{Na}$ tur aus eigenartige, aber auch strukturelle Bedeutungen. Die Aktionsarten gehören zu diesen strukturellen Aspekten; sie können nicht eigenartig sein.

Diese beiden Theorien befinden sich an der Grenze zwischen der Syntax und der logischen Form, an der heute so viel theoretisch gearbeitet wird.

\subsection{Kommentar}

Einen Theorievergleich hat es in der Geschichte der generativen Grammatik immer gegeben (Botha 1981). Wir haben gesehen, dass Idiome von Zeit zu Zeit als ein unterstützendes Argument für die eine oder andere Theorie verwendet wurden. Es ist auch klar, dass phraseologische Einheiten nur selten als Einheiten die zu erklären sind, betrachtet wurden. In diesem Sinn unterscheiden sich phraseologische Einheiten ganz von der anderen Bevölkerung des Lexikons, nämlich den Wörtern. In der generativen Theorie sind die Wörter etwa seit 1970 Objekt eines eigenen Forschungsbereiches, der Morphologie. Es gibt manche Lehrbücher der Morphologie (z. B. Spencer 1991), aber keines über die Phraseologie, zumindest nicht auf Englisch. Es existiert im Englischen nur ein Buch, das hier einen guten Überblick gibt (Wray 2002). Es ist auch verständlich, dass die Phraseologie in den Lehrbüchern der generativen Grammatik kaum eine Rolle spielt. In zwei sehr guten und gängigen Lehrbüchern kommt "Idiom“ im Stichwortverzeichnis entweder gar nicht vor (Haegeman 1991), oder es gibt nur fünf Zitate und fast alle haben mit der VP-Innensubjekt-Hypothese zu tun (Radford 1997). Morphologen interessieren sich grundsätzlich für lexikale Einheiten, wie sie existie- 
ren und wie Wissenschaft und Theorie mit existierenden Wörtern umzugehen haben. In der generativen Grammatik jedoch spielen phraseologische Einheiten nur eine untergeordnete Rolle. Es gibt kaum Theorien über die phraseologischen Einheiten selbst, und die generative Grammatik befasst sich nur mit Idiomen. Feste Kollokationen, Sprachformeln usw. sind nicht von primärem Interesse. Das wird den Lexikographen und den Soziolinguisten überlassen. Es gibt anscheinend wenig Interessantes über phraseologische Einheiten, mit Ausnahme der Idiome, und diese sind auch nur brauchbar als Beweis für andere Theorien.

Ein Beispiel dafür findet sich in einem kürzlich erschienenen Buch über lexikale Einheiten. „Die traditionelle Annahme ist, dass lexikale Einfügung aus dem Ersetzen eines $\mathrm{X}^{0}$ durch ein Wort besteht. Simultane Einfügung mehrerer Wörter, wie bei phrasalen Idiomen, ist wahrscheinlich außergewöhnlich", so der Herausgeber (Coopmans et al. 2000, S. ix). Wie kommt man zu einer solchen Auffassung? In keinem Kapitel des Buches sind phraseologische Einheiten und Idiome enthalten. Es ist auch nicht verwunderlich, dass in kaum einem Buch über generative Grammatik, in dem lexikale Einheiten behandelt werden, phraseologische Einheiten zu finden sind. Aber das bedeutet nicht, dass phraseologische Einheiten in der Syntax außergewöhnlich sind. Es gibt keine Forschungsarbeit, die das erwiesen hat. Ist dies eine statistische Behauptung oder eine Behauptung über Sprache oder Sprachgebrauch? Oder handelt es sich um die Aussage: „Wir interessieren uns nicht für die Möglichkeit, dass phraseologische Einheiten in der Grammatik einer Sprache eine Rolle spielen könnten.“

\section{Welche syntaktischen Eigenschaften phraseologischer Einheiten könnten aus der Sicht der generativen Grammatik interessant sein?}

\subsection{Einführung}

Wir haben betont, dass Idiome als Evidenz in generativen Theorien benutzt werden. Es werden stets zwei empirische Hypothesen angenommen: dass Teile einer phraseologischen Einheit Phrasen sind, und dass sie ununterbrochen sind. Man sollte jedoch zuerst fragen, wie eng die Teile idiomatischer Ausdrücke miteinander verbunden sind. Sind sie mehr oder weniger fest? Und was bedeutet das in der generativen Grammatik? Als Forschungsproblem spielt das kaum eine Rolle. Aber es ist möglich, hier Antwort zu geben. Es gibt mindestens 100000 phraseologische Einheiten im Englischen (Pawley/Syder 1983), vielleicht auch im Deutschen, und es gibt phraseologische Wörterbücher. Ganz wie in der Morphologie gibt es hier einen Forschungsbereich, allerdings ohne generative Forschung. Wenn wir wüssten, was die syntaktischen Eigenschaften phraseologischer Einheiten wären, könnten sie in die Syntax projektiert werden und auch bei der Theoriebildung eine Rolle spielen. 
Phraseologische Einheiten sind lexikalische Einheiten und haben deshalb eine phonologische, eine syntaktische und eine semantische Form. Sie haben meistens auch Gebrauchseigenschaften. Man weiß, wie man den Ausdruck gebrauchen kann, zum Beispiel Grüß Gott als Gruß - aber nicht überall. Auch hier hat die generative Grammatik nichts zu sagen. Aber Gebrauchseigenschaften sind ein Teil des lexikalischen Wissens. Diese Eigenschaften sind darum sprachwissenschaftlich zu erklären. Sie sind vielleicht nicht ein Teil der Universalgrammatik.

\subsection{Syntaktische Eigenschaften phraseologischer Einheiten}

Wo und wie soll man mit einer generativen Theorie von der Struktur phraseologischer Einheiten beginnen? Vielleicht mit der Hypothese, dass der „Kopf" einer phraseologische Einheit fixiert sein muss (Koopman/Sportiche 1991). Das bedeutet, dass das Wort im Kopfplatz bekannt sein muss. Aber um eine Phrase zu sein, muss auch noch mindestens ein zweites Wort fixiert werden. Das zweite Wort ist auch „Kopf" einer eigenen Phrase (van Gestel 1995). Diese zweite Phrase muss sich im maximalen Projektionsbereich des ersten Kopfes befinden (Kuiper/Everaert 2000). Das ergibt, syntaktisch gesehen, eine minimale phraseologische Einheit, zum Beispiel auf Pump. In der folgenden Struktur ist $X$ der fixierte Kopf der Phrase und $Y$ ist gleichfalls fixiert: $X$ bedeutet auf und Y Pump.

Bild 3.

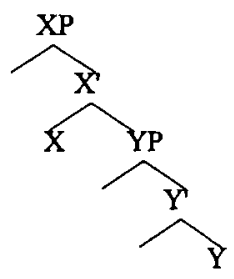

Bei längeren Phrasen wiederholt sich diese Konfiguration.

Das ganze ist eine Basistheorie über die Struktur phraseologischer Einheiten.

Vom Lexikon aus gesehen, erscheint die sich dadurch ergebende Struktur als das Resultat einer lexikalischen Wahl (Kuiper/Everaert 2000). Es hat sich indes gezeigt, dass die Köpfe eine syntaktische Auswahl bezüglich ihrer Komplemente treffen, und sie treffen auch eine semantische Wahl, zum Beispiel von menschlichen Subjekten. Augenscheinlich ist die phraseologische Fixierung das Resultat einer lexikalischen Wahl. Der Kopf einer Phrase wählt den Kopf einer weiteren Phrase in ihrem maximalen Projektionsbereich aus. Dieser „Unterkopf" kann dann einen weiteren Kopf wählen.

Man kann auch fragen, welche Konstituenten leer stehen können. Lexikale Köpfe können das nur selten, aber für Funktionsköpfe ist das anders. Zum 
Beispiel es ist möglich, dass in einer PP die Präposition und das Substantiv fixiert, das heißt lexikalisiert sind, aber der Determiner nicht. In das Idiom aus Erfahrung wissen beispielsweise könnten auch ein possessives NP oder ein Artikel eintreten; denn diese Stelle des Spezifikators von NP ist unbesetzt.

Es scheint, dass nach dieser Theorie phraseologische Einheiten eigentlich nicht Phrasen, sondern vielmehr die Phrasen das Resultat der lexikalischen Wahl eines Kopfes sind. Eine solche Wahl ist beschränkt. Lexikale Köpfe müssen ausgewählt werden, und sie müssen sich im maximalen Projektionsbereich befinden.

Einige weitere Folgerungen sind möglich. Alle Wahleigenschaften eines Kopfes können ihre Wirkung haben. Wenn dem so ist, dann sind unbesetzte Konstituenten notwendigerweise immer das Resultat einer Konstituentenwahl des Kopfes. Das scheint möglich zu sein, ebenso aber, dass die Konstituentenwahl für Idiome aufgehoben ist. Die semantischen Wahleigenschaften des Kopfes können in Idiomen vorbestimmt sein. Kollokationen können aber den semantischen und syntaktischen Wahleigenschaften des Kopfes folgen.

Mit diesen potentiellen Theorien wollte ich darlegen, dass generative Theorien, welche die syntaktischen Eigenschaften phraseologischer Einheiten mit einbeziehen, formuliert werden können.

\section{Schluss}

Es gibt keinen Forschungsbereich in der generativen Grammatik, der sich mit den Eigenschaften phraseologischer Einheiten befasst. Idiome werden als Fakten in Auseinandersetzungen hier und da benutzt, aber ohne dass man sich die Mühe machen würde, sie zu betrachten und ihnen einen dementsprechenden Platz einzuräumen. Man nimmt an, dass Idiome nicht unterbrochen sind, allein weil das im speziellen Sinn der Fall ist. Wahrscheinlich ist noch viel zu tun, um einen theoretisch interessanten Forschungsbereich zu entwickeln, der sich damit befasst, wie phraseologische Einheiten in der generativen Grammatik behandelt werden können. Man braucht für ein solches Vorhaben den Nachweis, dass phraseologische Einheiten, wie auch Wörter, einen eigenen Forschungsbereich darstellen können, und, dass der Forschungsbereich Phraseologie dann, und nur dann, zur generativen Sprachtheorie beitragen kann.

\section{Literatur}

Becker, Joseph (1975): The phrasal lexicon. In: Shank, Roger/Nash-Webber, B. L. (Hg.): Theoretical Issues in Natural Language Processing. Cambridge, Massachusetts. S. $60-63$.

Botha, Rudolf P. (1981): The conduct of linguistic inquiry: A systematic introduction to the methodology of generative grammar. The Hague.

Bresnan, Joan (Hg.) (1982): The mental representation of grammatical relations. Cambridge, Massachusetts. 
Chafe, Wallace L. (1968): Idiomaticity as an anomaly in the Chomskian paradigm. In: Foundations of Language, 4, S. 109-127.

Chomsky, Noam (1965): Aspects of the theory of syntax. Cambridge, Massachusetts.

Chomsky, Noam (1970): Remarks on Nominalizations. In: Jacobs, Roderick A./Rosenbaum, Peter S. (Hg.): Readings in English Transformational Grammar. Waltham, Massachusetts. S. 184-221.

Chomsky, Noam (1975): The logical structure of linguistic theory. Chicago.

Chomsky, Noam (1981): Lectures on government and binding. Dordrecht.

Chomsky, Noam (1986a): Barriers. Cambridge, Massachusetts.

Chomsky, Noam (1986b): Knowledge of language: its nature, origin and use. New York.

Chomsky, Noam (1996): Minimalism. Cambridge, Massachusetts.

Coopmans, Peter/Everaert, Martin/Grimshaw, Jane (2000): Introduction. In: Coopmans, Peter/Everaert, Martin/Grimshaw, Jane (Hg.): Lexical specification and insertion. Amsterdam/Philadelphia. S. ix-xvii.

de Saussure, Ferdinand (1959): Course in general linguistics. New York.

Dobrovol'skij, Dmitrij (2000): Ist die semantik von idiomen nicht compositionell? In: Beckman, Suzanne/König, Peter-Paul/Wolf, Georg, S. (Hg.): Sprachspiel und Bedeutung. Festschrift für Franz Hundsnurscher zum 65. Geburtstag. Tübingen. S. 113-124.

Everaert, Martin/Kuiper, Koenraad (1996): Theory and data in idiom research. Paper presented at Parasession on Theory and Data in Linguistics, Chicago.

Fraser, Bruce (1970): Idioms within a transformational grammar. In: Foundations of language, 6, S. 22-42.

Haegeman, Liliane (1991): Introduction to government and binding theory. Oxford.

Hale, Ken/Keyser, Jay (1998): The basic elements of argument structure. In: MIT Working Papers in Linguistics, 32, S. 73-118.

Halle, Morris (1973): Prolegomena to a theory of word formation. In: Linguistic Inquiry, 4, S. 3-16.

Harris, Randy Allen (1995): The linguistics wars. Oxford.

Jackendoff, Ray S. (1975): Morphological and semantic regularities in the lexicon. In: Language, 51, S. 639-671.

Koopman, Hilda/Sportiche, Dominique (1991): The position of subjects. In: Lingua, 85, S. 211-258.

Kuiper, Koenraad/Everaert, Martin (2000): Constraints on the phrase structural properties of English phrasal lexical items. In: PASE papers in language studies: Proceedings of the 8th annual conference of the Polish Association for the study of English, S. 151-170. Wroclaw.

Larson, Richard R. (1988): On the double object construction. In: Linguistic Inquiry, 19, S. 335-391.

Levine, Robert (1984): Against reanalysis rules. In: Linguistic Analysis, 14, S. 3-29.

Marantz, Alec (1984): On the nature of grammatical relations. Cambridge, Massachusetts.

McGinnis, Martha (2002): On the systematic aspect of idioms. In: Linguistic Inquiry, 33, S. $665-672$.

Nunberg, Geoffrey/Sag, Ivan/Wasow, Tom (1994): Idioms. In: Language, 70, S. 491-538.

Pawley, Andrew/Syder, Frances (1983): Two puzzles for linguistic theory: nativelike selection and nativelike fluency. In: Richards, Jack/Schmidt, Richard (Hg.): Language and Communication. London. S. 191-226.

Pollard, C./Sag, Ivan (1988): Information-based syntax and semantics (Vol.1). Stanford.

Radford, Andrew (1997): Syntactic theory and the structure of English. Cambridge. 
Richards, Norvin (2001): An idiomatic argument for lexical decomposition. In Linguistic Inquiry, 33, S. 183-192.

Spencer, Andrew (1991): Morphological theory. Oxford.

van Gestel, Frank (1995): En bloc insertion. In: Everaert, Martin/van der Linden, EricJan/Schenk, André/Schroeder, Rob. (Hg.): Idioms: structural and psychological perspectives. Hillsdale, New Jersey. S. 75-94.

Weinreich, Uriel (1969): Problems in the analysis of idioms. In: Puhvel, Jaan (Hg.): Substance and the Structure of Language. Berkeley. S. 23-81.

Wray, Alison (2002): Formulaic language and the lexicon. Cambridge. 